\title{
Prevalence and Modifiable Risk Factors of Non- Communicable Diseases among Jakun Orang Asli at Tasik Chini, Pekan, Pahang.
}

\author{
Wong $C M^{a}$, Faiz $D^{a}$, Diana Safraa $S^{a}$, Raja Mohd Azim $R H^{b}$, Siti Zubaidah $A R^{c}$ \\ ${ }^{a}$ Health Promotion Unit, Department of Community Medicine, Faculty of Medicine UKM, \\ Universiti Kebangsaan Malaysia Medical Center. \\ ${ }^{b}$ District Health Office Batang Padang, Perak. \\ ${ }^{c}$ Non-communicable Disease Unit, Pahang State Health Department
}

\section{ABSTRACT}

Introduction: Non-communicable disease accounted for 73\% of premature death in year 2015 Malaysian national survey. The orang asli population may be affected similarly. The prevention of non-communicable diseases should start as early as modifiable risk factors prevention, as proposed by STEPWISE approach of WHO. This study aims to identify the prevalence of hypertension, diabetes mellitus and dyslipidaemia among Jakun orang asli population and examine the association with risk factors. Methods: This is a cross-sectional study analysing 72 case reports of General health screening done in year 2015. The study population was adult Jakun orang asli Tasik Chini using cluster sampling followed by simple random sampling methods. Chi Square test was used for bivariate analysis of relationship each variable has with the diseases, and binary logistic regression was used to analyse association of covariates with each disease. Results: The prevalence was $41.7 \%$ for hypertension, $25 \%$ for Diabetes mellitus, $6.9 \%$ for dyslipidaemia. Education level was significantly related to hypertension, $X^{2}=11.565$ (1), $p=0.001$; obesity was significantly related to diabetes, $X^{2}=8.333$ (1), $p=0.004$. After adjusted for covariates, low education level has 13.379 odds of getting hypertension. Obesity has 7.384 odds of getting diabetes mellitus; female gender has higher odds of getting dyslipidaemia while younger age, physically active and not smoking are protective factors. Conclusion: Higher prevalence of hypertension and diabetes mellitus was found among Tasik Chini orang asli population. Lower sociodemographic characteristics and unhealthy lifestyle factors are associated with the diseases.

KEYWORDS: Non-communicable diseases, Orang Asli, Jakun, modifiable risk factor, obesity

\section{INTRODUCTION}

Modernization has shifted the disease burden focus to non-communicable diseases. ${ }^{1-3}$ The prevalence of non-communicable diseases has been in steep uprising trend for the past 10 years globally and regionally ${ }^{4}$, recent local Malaysian studies have been consistent in this uprising trend too. Noncommunicable diseases contributed to $73 \%$ of

Corresponding author:

Dr. Faiz (Daud)

Department of Community Medicine,

Faculty of Medicine UKM,

Universiti Kebangsaan Malaysia Medical Center, Jalan Yaacob Latif, Bandar Tun Razak, 56000 Batu 9 Cheras,

Wilayah Persekutuan Kuala Lumpur, Malaysia

Tel: 03-9145 5903 Fax: 03-9145 6670

Email:drfaizdaud@gmail.com mortality rate in Malaysia, with diabetes, obesity and hypertension among the top three attributable risk factors to Disability Adjusted Life Years. ${ }^{5-9}$ Noncommunicable diseases prevention is one of the major focuses of Sustainable Developmental Goal (SDG), because the four leading non-communicable diseases have contributed to 14 million premature deaths worldwide annually. ${ }^{1,10}$ Therefore, the focus of addressing non-communicable diseases is no longer at treating and preventing the diseases, but aiming to prevent the development of risk factors adopting WHO STEPSwise approach (a World Health Organization recommended theoretical framework on the surveillance of non-communicable Diseases) since year 2005..$^{2,11,12}$ WHO has committed to reduce $25 \%$ of premature death from these noncommunicable diseases by the year of 2025 in order to prevent 7 trillion USD losses of country 
productivity to healthcare expense. In year 2013, World Health Organization has devised the Global Action Plan for non-communicable diseases for year 2013-2020. ${ }^{13}$

From the last national validated data, Malaysia ranked top obese population (76\%) in South-east Asia and Western-pacific region. ${ }^{3,13,14}$ The younger age of onset of obese population is alarming. Malaysian are trending towards unhealthy lifestyles, adopting more health risk behaviours like smoking, alcohol consumption, low dietary fiber intake and physical inactivity. ${ }^{6,10,15}$ Therefore, sociodemographic factors, anthropometrical factors and lifestyle factors are hypothesized to have relationship with hypertension, diabetes mellitus and dyslipidaemia.

In recent years, urbanization in Malaysia has changed the way the local aborigines live and interact with their non-aborigine counterparts, caused them to have similar accessibility to food source, the resettlement to brick house at urban fringe changed the way they move aro ${ }^{16-18}$ Recent study shows indigenous around the world are experiencing similar, if not more drastic rise in the prevalence of non-communicable diseases. ${ }^{19}$ Jakun is the second largest Malaysia aboriginal sub-tribe Proto-Melayu- who shared same Taiwan ancestry and Yunan origin, they speak proto-Austroneisan. ${ }^{20}$, 21 They have much higher perinatal mortality rate with much lower life expectancy at birth, displaced below the hard-core poverty line of Malaysians. ${ }^{17,}{ }^{20}$, 22 In the studies of Ngui et.al, ${ }^{22} 90 \%$ of the Jakun population are not working, the rest are gathering forestry products.

Amongst the many researches on prevalence of noncommunicable diseases, only few were done among the Malaysian aborigines, no local available centralized data on aboriginal non-communicable diseases status as they only account for $0.5 \%$ of the Malaysian population. ${ }^{23}$ Few studies examined the association of modifiable risk factors but rarely combining all the main lifestyle factors in association study, particularly in the orang asli community. This paper provided the prevalence of the three common non-communicable diseases among the Jakun, the main sub-tribe of orang asli in Malaysia which is the proxy to cardiovascular disease. Subsequently, the association of each sociodemographic, anthropometrical and lifestyle factor was tested against the three diseases independently and in combined model; confounders adjusted to produce attributable risk of each factor towards Tasik Chini Jakun population, one of the few homogenous aboriginal communities in Peninsular Malaysia, which has gained much global attention due to the pollution and urban-rural interaction of this UNESCO Biosphere reserve site. To the principal author's best knowledge, there is no prior study on the effect of urbanization on Peninsular orang asli population on the prevalence of non-communicable diseases.

\section{METHODOLOGY}

This is a cross-sectional study analysing case report of available primary data set on non-communicable diseases screening done by Raub and Pekan District Health Office using the Bahasa Melayu version (the Jakun's conversational language) validated general health screening form used by Malaysia Ministry of Health in year 2015, followed by anthropometrical assessments and capillary blood glucose, blood cholesterol measurements done using calibrated machines by skilled medical personnel. The study was carried out in six months from February to August 2018 to identify the prevalence of hypertension, diabetes mellitus and dyslipidaemia and their associated risk factors among the Jakun tribe orang asli settlement surrounding tasik (lake) Chini, Pekan, Pahang. The study was conducted in six villages surrounding the Tasik Chini, namely Kampung Cendahan, Kampung Tanjung Puput, Kampung Gumum, Kampung Melai, Kampung Ulu Melai, Kampung Ulu Gumum with a coverage area of $69.5 \mathrm{~km}^{2,24}$.

This study population was all orang asli in Tasik Chini, sampling frame was all adult with local residential address who met all criteria, consented and completed questionnaire and clinical assessment. Sampling method was done in twostages using cluster sampling method followed by simple random sampling method (figure 1). The inclusion criteria are: 1. Adult of both male and female genders age 18 year old or above; 2 . residence around Tasik Chini where house of interview belongs to him. Exclusion criteria are: 1. Pregnancy or post-natal period within 42 days; 2. any individual not compatible mentally to give consent or to be interviewed verbally. A sample size of 72 was collected from the case report, with 
respond rate of $100 \%$ from all subjects who met the screening criteria. Based on the household survey of Tasik Chini Jakun population in year 2015, only quarter of the residents were adult (125 subjects), sample size calculation using the highest prevalence among the three diseases studied $\mathbf{3 0 . 8 \%}$ for hypertension) yielded $79 \%$ power of study (openEpi 2013). ${ }^{47}$

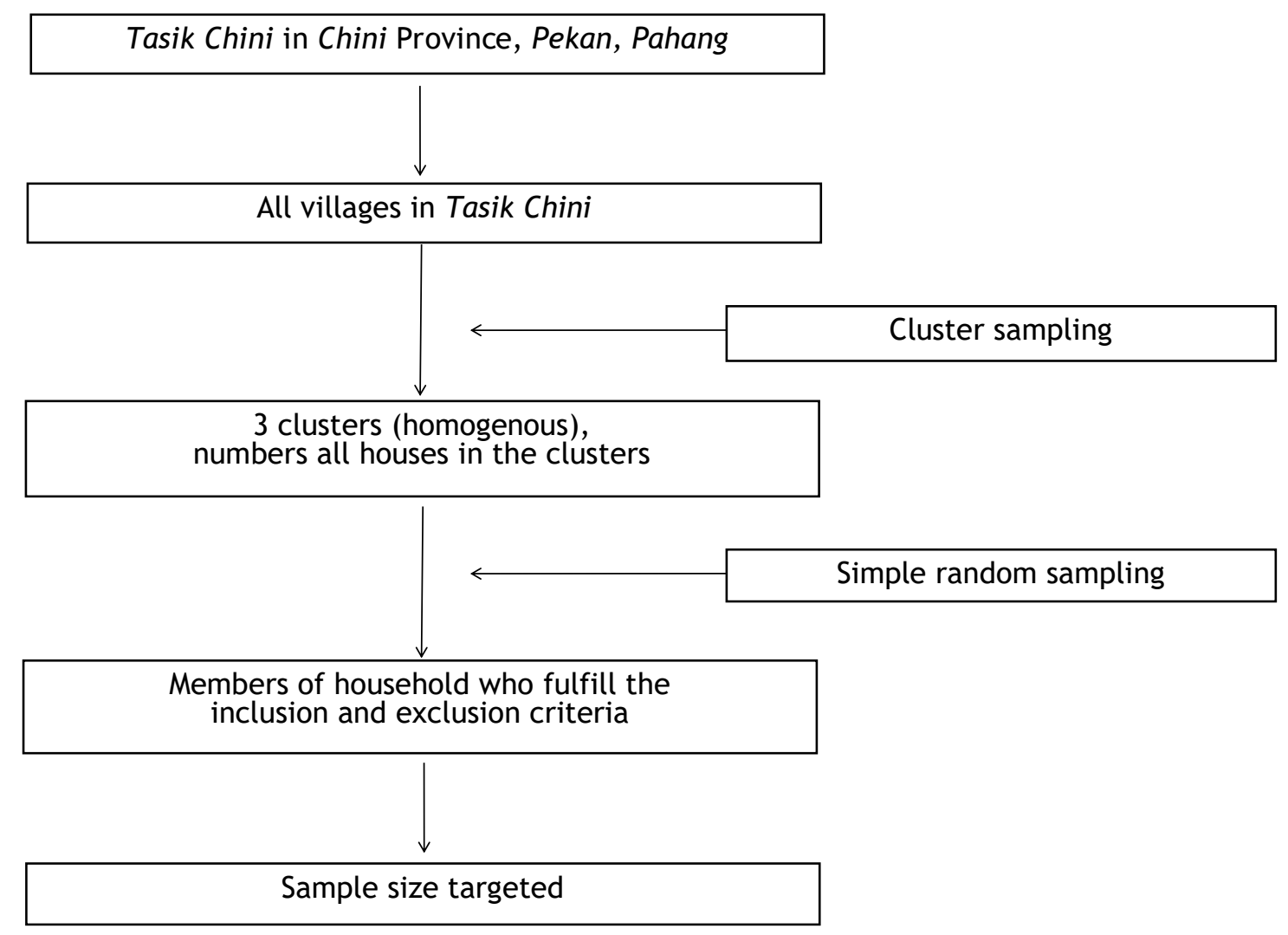

Figure I: Flow of Sampling

Body mass index was classified based on clinical practice Guideline on obesity 2004 for the purpose of comparison with the NHMS 2015 prevalence. ${ }^{6,25-27}$ Hypertension is defined as diagnosis made by medical personnel at least four weeks prior to date of survey. Two abnormal readings of blood pressure (above $140 / 90 \mathrm{mmHg}$ ) with adequate rest was considered as newly diagnosed hypertension. Blood cholesterol was screened using Cardiocheck capillary cholesterol meter. Dyslipidaemia is defined as a reading of $\geq 5.2 \mathrm{mg} / \mathrm{dL}$. Capillary blood glucose was screened using Accucheck glucometer, followed $4^{\text {th }}$ edition Clinical Practice Guideline on Diabetes Mellitus, adopting the methodology of National Health and Morbidity Survey 2015 as defined by WHO using capillary blood. 6, 28, 29 Normal fasting blood glucose (FBG, $<5.6 \mathrm{mmol} / \mathrm{l}$ ), Impaired Fasting Glucose (IFG, fasting blood glucose between $\geq 5.6$ and $<6.1 \mathrm{mmol} / \mathrm{l}$ ), Impaired Glucose Tolerance (IGT, fasting blood glucose between $\geq 7.8$ and $<11.0$ $\mathrm{mmol} / \mathrm{l}$ ), newly diagnosed diabetes (fasting blood glucose $\geq 7 \mathrm{mmol} /$ or random blood glucose $\geq 11.1$ $\mathrm{mmol} / \mathrm{l})$. Diabetes mellitus is any respondent on anti -diabetic or diagnosis made by medical personnel at least four weeks prior to date of survey.

\section{Data Analysis}

Data entry and analysis was done using Predictive Analytics Software, the Statistic Program for Social

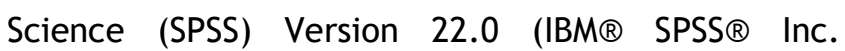
Released 2013) as descriptive, bivariate and multivariate analyses. Normality of data distribution was checked. Continuous variables with normal distribution were reported in mean $\pm 2 \mathrm{SD}$, while median was reported up to one decimal point for abnormal distribution. Frequency and percentage were used for categorical variables. Discrete data and proportions, proportion odd ratios were tested for significance using Pearson's Chi-square, $\mathrm{X}^{2}$, with confidence interval of $95 \%$, $p$ value of $<0.05$ was set as significant value, power of study set as $80 \%$. Independent variables were tested by simple logistic regression; confounders were adjusted in multiplelogistic regression analysis to determine association. Results reported as prevalence Odds ratio (OR) with 
95\% confidence interval. Model was tested for stability and interaction. Results were analysed in the consideration of possible biases.

\section{RESULTS}

\section{Demographic Characteristics}

Table I listed the clinical characteristics of the studied population, $26.4 \%$ had 2 cardiovascular risk factors, $47.2 \%$ had 3 risk factors and $4.2 \%$ had 4 risk factors. Table II shows the prevalence of hypertension, blood glucose level, blood cholesterol level and BMI and their relationships with the sociodemographic factors and anthropometric risk factors. The prevalence of hypertension was $41.7 \%$ (9.7\% known hypertension, $31.9 \%$ newly diagnosed, $11.1 \%$ uncontrolled); prevalence of diabetes mellitus was $25 \%, 4.2 \%$ known case, $18.1 \%$ newly diagnosed IGT, 2.7\% newly diagnosed DM; prevalence of dyslipidaemia was $6.9 \%$, all new diagnosed.

\section{Prevalence of Non-communicable Diseases}

Hypertension increases significantly with age $(\mathrm{p}=$ $0.0056, \mathrm{OR}=3.171,95 \% \mathrm{Cl}$ OR 0.939; 10.713), but inverse with education level $86.7 \%(p=0.001, O R=$ 7.15, 95\% Cl OR 2.123, 24.083). Median RBS was $6.8 \mathrm{mmol} / \mathrm{dl}, 66.7 \%$ of them had poor glycaemic control; $95.8 \%$ of the respondents never had capillary glucose checked before. Only obesity has significant higher prevalence of diabetes mellitus $(p=0.004,95 \%$ OR $=3.956 ; 95 \% \mathrm{Cl}$ OR 1.594, 15.412). All lifestyle risk factors related to prevalence of diabetes in theoretical manner but all were insignificantly related.

Subjects with higher diabetic range and impaired glucose tolerance reading did not appear to have higher SBP or DBP. The prevalence of obesity was $33.3 \%$ by $\mathrm{BMI}$ and waist circumference; median capillary cholesterol was $3.36 \mathrm{mg}$. Subjects with unhealthy lifestyle factors were found to have higher BMI, but none are found significant related to higher prevalence of obesity $(p=>0.05)$. Nondrinker has $4 \mathrm{x}$ times higher odd of dyslipidaemia ( $\mathrm{p}$ $=0.111, \mathrm{OR}=4.296,95 \% \mathrm{Cl}$ OR 0.629, 29.365); nonsmoking showed much lower odd of developing dyslipidaemia $(\mathrm{OR}=0.169,95 \% \mathrm{Cl} \mathrm{OR}=0.018$, 1.593, $p=0.084$ ).

\section{Association of Risks Factors with Various Non- Communicable Diseases}

Table III shows simple logistic regression analysis of individual risk factor in association to hypertension, diabetes mellitus and dyslipidaemia while table IV summarized factors significant for each of the three non-communicable diseases with covariates adjusted. Age, education level and marital status were associated with hypertension, with odd ratios highest for education level, persisted after adjusted for all other covariates $(\operatorname{adj} \mathrm{OR}=13.379,95 \% \mathrm{Cl}$ OR $3.158 ; 56.656, X^{2}=16.485$ (1), p $0.000(<0.05)$, no interaction effect was tested from all the significant covariates. For diabetes mellitus, only obesity is significantly associated, persisted after adjustment $\left(\operatorname{adj} \mathrm{OR}=7.384, \mathrm{Cl} 95 \%\right.$ adj OR 1.895; 28.767, $X^{2}=$ 9.524 (1), $p=0.002$ ). Dyslipidaemia was discussed at bivariate level using Fisher's exact test due to small sample size.

\section{DISCUSSION}

The prevalence of hypertension and diabetes mellitus and obesity were higher in the Tasik Chini Jakun orang asli population as compared with NHMS $2015 .^{30}$ Almost half of the studied population has more than 3 risk factors for the development of cardiovascular diseases, majority were newly diagnosed, while poor control for known cases, reflecting the rising endemicity of common noncommunicable diseases and under-utilization of the healthcare services in this population.

The outliers caused skewness of distribution at extreme ends of blood pressure, blood glucose and blood cholesterol reading. Similar cross-sectional study in the same location 3 years prior to this study showed much lower prevalence for all three diseases, but no detail methodology on screening was mentioned, result stopped at descriptive analysis.

Age was significantly related to hypertension as similar to findings in many previous studies ${ }^{31,32}$, coherent with chronic nature of non-communicable diseases, degenerative loss of protective hormones, dysregulation of homeostasis. ${ }^{31-33}$ Faiz et.al found much higher prevalence of hypertension in local clinic subjects, this may possibly bias towards sampling population. ${ }^{32}$ Low education level 
attributed $92.5 \%$ of hypertension in this population. In Henry J.P (1988) observation of rural and urban blood pressure, the rise of blood pressure was marked in low salt diet urban population with neuroendocrine arousal due to stress. ${ }^{33,34}$ Lower stress reserve group faces higher neuro-endocrinal arousal. ${ }^{35}$ Mente et.al in contrast found steeper rise in blood pressure in association with salt intake for hypertensive. ${ }^{36}$ Lifestyle factors further increase the peripheral resistance by atherosclerosis, arteriolosclerosis, endothelial injury cascade activation.
For the second non-communicable disease studied here, the prevalence of Diabetes Mellitus and Impaired Glucose Tolerance reported drastic rise when evaluated against a study done 3 years back in the same population. ${ }^{63}$ Obesity attributed to $86.5 \%$ of diabetes mellitus. The relationship of obesity and diabetes mellitus are well established in previous studies, via the pathway of insulin resistance, and the sharing pathogenesis for liver fat metabolism dysfunction. ${ }^{37}$ Obese females of lower occupation energy level have significant higher glycaemic readings.

Table I: Characteristics of studied population

\begin{tabular}{|c|c|c|c|}
\hline \multicolumn{2}{|c|}{ Variable } & $\mathbf{N}$ & Percentage \% \\
\hline \multicolumn{4}{|c|}{ Sociodemographic factors } \\
\hline \multicolumn{4}{|l|}{ Age group } \\
\hline & adult & 58 & 80.6 \\
\hline & $18-40$ & 37 & 51.4 \\
\hline & $41-59$ & 21 & 29.2 \\
\hline & elderly & & \\
\hline & 60 and above & T- & 17.4 \\
\hline Median age $(y$ & & 39.5 & \\
\hline \multicolumn{4}{|l|}{ Gender } \\
\hline & Male & 28 & $38.90 \%$ \\
\hline & Female & 42 & $61.60 \%$ \\
\hline \multicolumn{4}{|c|}{ Educational Level } \\
\hline & Low & 46 & $63.90 \%$ \\
\hline & No formal school & 30 & $41.70 \%$ \\
\hline & Primary school & 16 & $22.20 \%$ \\
\hline & High & 26 & $36.10 \%$ \\
\hline & Secondary school & 25 & $34.70 \%$ \\
\hline & Tertiary education & 1 & $1.40 \%$ \\
\hline \multicolumn{4}{|c|}{ Occupational Energy level } \\
\hline & Low & 40 & 55.6 \\
\hline & Executive & 1 & 1.4 \\
\hline & Businessman & 3 & 4.2 \\
\hline & Unemployed & 9 & 12.5 \\
\hline & housewives & 27 & 37.5 \\
\hline & High & 32 & 44.4 \\
\hline & Fisherman/boatman & 10 & 13.9 \\
\hline & Odd jobs (collect forestry products) & 22 & 30.6 \\
\hline \multirow{4}{*}{ Marital Status } & Married & 56 & 77.8 \\
\hline & Not married & 16 & 22.2 \\
\hline & Single & 10 & 13.9 \\
\hline & Divorced & 6 & 8.3 \\
\hline \multicolumn{4}{|c|}{ Anthropometrical factors } \\
\hline Height (m) & Median & 1.5 & \\
\hline Weight (kg) & median & 51.6 & \\
\hline
\end{tabular}




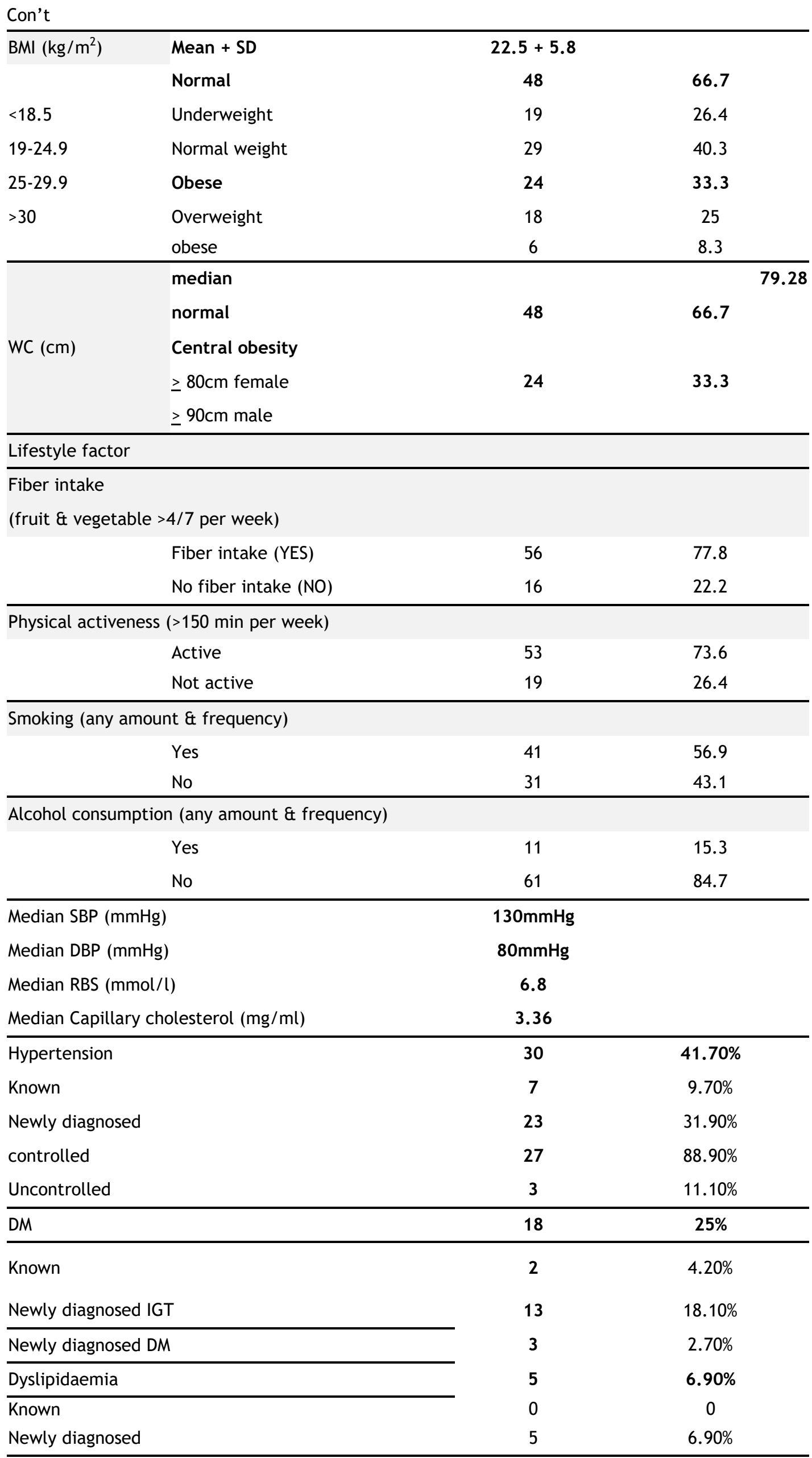


Table II: Prevalence and risk estimate of blood pressure level, blood glucose level and blood cholesterol level in relation to socio-demographic factors, anthropometrical factors and lifestyle factors.

\begin{tabular}{|c|c|c|c|c|c|c|c|c|c|c|c|c|}
\hline Factors & $\begin{array}{l}\text { HPT } \\
(\mathrm{n}=30) \\
\text { Prevalence } \\
(\%)\end{array}$ & $x^{2}$ (df) & $\mathbf{P}$ & OR $(95 \% \mathrm{Cl})$ & $\begin{array}{l}D M \\
(n=18) \\
\text { Prevalence (\%) }\end{array}$ & $X^{2}(d f)$ & $\mathbf{P}$ & OR $(95 \% \mathrm{Cl})$ & $\begin{array}{l}\text { Dyslipidaemia } \\
(n=5) \\
\text { Prevalence } \\
(\%)\end{array}$ & $X^{2}(d f)$ & $\mathbf{P}$ & OR $(95 \% \mathrm{Cl})$ \\
\hline \multicolumn{13}{|c|}{ Socio-demographic risk factor (s) } \\
\hline \multicolumn{13}{|c|}{ Age group } \\
\hline Adult & $21(70 \%)$ & $\begin{array}{c}16.224 \\
(2)\end{array}$ & $\begin{array}{c}0.000 \\
(<0.05)\end{array}$ & $\begin{array}{c}3.171(0.939 \\
10.713)\end{array}$ & $15(80.6 \%)$ & $0.118(1)$ & 0.731 & $\begin{array}{c}0.782(0.192 \\
3.188)\end{array}$ & $5(100 \%)$ & $\begin{array}{l}1.297 \\
(1)\end{array}$ & 0.255 & $\begin{array}{c}0.791(0.699 \\
0.895)\end{array}$ \\
\hline $\begin{array}{l}(18-40 \\
\text { years }) \\
\end{array}$ & $7 \quad(23.3 \%)$ & & & & $8(44.4 \%)$ & & & & 0 & & & \\
\hline $\begin{array}{l}(41-59 \\
\text { years })\end{array}$ & $14(46.7 \%)$ & & & & $7(38.9 \%)$ & & & & $5(100 \%)$ & & & \\
\hline $\begin{array}{l}\text { Elderly } \\
(60 \\
\text { years } \\
\text { and } \\
\text { above) } \\
\end{array}$ & $9(30 \%)$ & 5 & & & $3(19.4 \%)$ & 11 & & & $0(0 \%)$ & & & \\
\hline \multicolumn{13}{|l|}{ Gender } \\
\hline Male & $13(43.3 \%)$ & $\begin{array}{l}0.427 \\
(1)\end{array}$ & 0.513 & $\begin{array}{c}1.134(0.528 \\
3.592)\end{array}$ & $5(27.8 \%)$ & $1.247(1)$ & 0.264 & $\begin{array}{c}0.518(0.162 \\
1.660)\end{array}$ & $3(60 \%)$ & $\begin{array}{l}1.008 \\
(1)\end{array}$ & $0.293^{\mathrm{a}}$ & $\begin{array}{l}2.52(0.394 \\
16.130)\end{array}$ \\
\hline Female & $17(56.7 \%)$ & & & & $13(72.7 \%)$ & & & & $2(40 \%)$ & & & \\
\hline \multicolumn{13}{|c|}{ Education level } \\
\hline Low & $26(86.7 \%)$ & $\begin{array}{c}11.565 \\
(1)\end{array}$ & 0.001 & $\begin{array}{c}7.150(2.1233 \\
24.083)\end{array}$ & $11(61.1 \%)$ & $0.080(1)$ & 0.777 & $\begin{array}{c}0.853(0.284 \\
2.563)\end{array}$ & $4(80 \%)$ & $\begin{array}{c}0.605 \\
(1)\end{array}$ & 0.437 & $\begin{array}{l}2.381(0.252 \\
22.512)\end{array}$ \\
\hline High & $4 \quad(13.8 \%)$ & & & & $7 \quad(38.9 \%)$ & & & & $1(20 \%)$ & & & \\
\hline \multicolumn{13}{|c|}{ Job energy level } \\
\hline Low & $13(43.3 \%)$ & $\begin{array}{c}3.111 \\
(1)\end{array}$ & 0.078 & $\begin{array}{c}0.425(0.163 \\
1.109)\end{array}$ & $8(44.4 \%)$ & $1.2(1)$ & 0.273 & $\begin{array}{c}0.55(0.187 \\
1.614)\end{array}$ & $4(80 \%)$ & $1.3(1)$ & 0.254 & $\begin{array}{c}3.444(0.365 \\
32.462)\end{array}$ \\
\hline High & $17(56.7 \%)$ & & & & $10(55.6 \%)$ & & & & $1(20 \%)$ & & & \\
\hline \multicolumn{13}{|c|}{ Marital status } \\
\hline Married & $25(83.3 \%)$ & $\begin{array}{c}1.375 \\
(1)\end{array}$ & 0.241 & $\begin{array}{c}2.000(0.620 \\
6.447)\end{array}$ & $15(83.3 \%)$ & $0.642(1)$ & 0.423 & $\begin{array}{l}1.75(0.44 \\
\quad 6.963)\end{array}$ & $4(80 \%)$ & $\begin{array}{c}0.039 \\
(1)\end{array}$ & 0.844 & $\begin{array}{c}1.255(0.131 \\
12.051)\end{array}$ \\
\hline \begin{tabular}{l|} 
Not \\
married
\end{tabular} & $5 \quad(16.7 \%)$ & & & & $3(16.7 \%)$ & & & & $1(20 \%)$ & & & \\
\hline \multicolumn{13}{|c|}{ Anthropometrical risk factor (s) } \\
\hline \multicolumn{13}{|l|}{ Obesity } \\
\hline $\begin{array}{l}\text { Non } \\
\text { Obese }\end{array}$ & $18(60 \%)$ & $\begin{array}{c}1.029 \\
(1)\end{array}$ & 0.31 & $\begin{array}{c}1.667(0.619 \\
4.489)\end{array}$ & $7(38.9 \%)$ & $8.333(1)$ & 0.004 & $\begin{array}{c}3.956(1.594 \\
15.412)\end{array}$ & $2(40 \%)$ & $\begin{array}{c}1.719 \\
(1)\end{array}$ & 0.19 & $\begin{array}{c}3.2286(0.51 \\
21.152)\end{array}$ \\
\hline Obese & $12(40 \%)$ & & & & $11(61.1 \%)$ & & & & $3(60 \%)$ & & & \\
\hline \multicolumn{13}{|c|}{ Lifestyle risk factor (s) } \\
\hline \multicolumn{13}{|c|}{ Fiber intake } \\
\hline Yes & $23(76.7 \%)$ & $\begin{array}{c}0.037 \\
(1)\end{array}$ & 0.848 & $\begin{array}{c}1.116(0.363 \\
\quad 3.427)\end{array}$ & $13(72.2 \%)$ & $0.429(1)$ & 0.513 & $\begin{array}{c}1.503(0.441 \\
5.122)\end{array}$ & $5(100 \%)$ & $\begin{array}{c}1.535 \\
(1)\end{array}$ & 0.215 & $\begin{array}{c}0.761(0.666 \\
0.87)\end{array}$ \\
\hline No & $7 \quad(23.3 \%)$ & & & & $5 \quad(27.8 \%)$ & & & & $0(0 \%)$ & & & \\
\hline \multicolumn{13}{|c|}{ Physical active } \\
\hline yes & $24(80 \%)$ & $\begin{array}{c}1.081 \\
(1)\end{array}$ & 0.299 & $\begin{array}{c}0.558(0.184 \\
1.689)\end{array}$ & $12(66.7 \%)$ & $0.596(1)$ & 0.44 & $\begin{array}{c}1.577(0.494 \\
5.039)\end{array}$ & $5(100 \%)$ & $\begin{array}{c}1.926 \\
(1)\end{array}$ & 0.165 & $\begin{array}{c}0.716(0.616 \\
0.833)\end{array}$ \\
\hline No & $6(20 \%)$ & & & & $\begin{array}{ll}6 & (33.3 \%) \\
\end{array}$ & & & & $0(0 \%)$ & & & \\
\hline \multicolumn{13}{|l|}{ Smoking } \\
\hline Yes & $17(43.3 \%)$ & $\begin{array}{c}0.002 \\
(1)\end{array}$ & 0.968 & $\begin{array}{l}0.981(0.381 \\
2.526)\end{array}$ & $7(38.9 \%)$ & $3.1911(1)$ & 0.074 & $\begin{array}{c}0.374(0.125 \\
1.121)\end{array}$ & $1(20 \%)$ & $\begin{array}{l}2.991 \\
(1)\end{array}$ & 0.084 & $\begin{array}{c}0.169,(0.018 \\
1.593)\end{array}$ \\
\hline No & $13(56.7 \%)$ & & & & $11(61.1 \%)$ & & & & $4(80 \%)$ & & & \\
\hline \multicolumn{13}{|c|}{ Alcohol consumption } \\
\hline Yes & $4 \quad(13.3 \%)$ & $\begin{array}{c}0.150 \\
(1)\end{array}$ & 0.698 & $\begin{array}{l}0.769(0.204 \\
2.906)\end{array}$ & $1(5.6 \%)$ & $1.753(1)$ & 0.186 & $\begin{array}{c}0.259(0.031 \\
2.179)\end{array}$ & $2(40 \%)$ & $\begin{array}{c}2.537 \\
(1)\end{array}$ & 0.111 & $\begin{array}{c}4.296(0.629 \\
29.365)\end{array}$ \\
\hline No & $26(86.7 \%)$ & & & & $17(94.4 \%)$ & & & & $3(60 \%)$ & & & \\
\hline
\end{tabular}

The change of occupation role or lifestyle of the Jakun orang asli plays an important effect on the rising prevalence of diabetes mellitus. Recent year iron ore mining and deforestation at Bukit Chini have changed the sociodemographic characteristics of the Jakun orang asli, they have decreased physical activities related to collection of forestry and fishery products as the hill was cleared up and lake was polluted. ${ }^{24,38,39}$ The urban-rural interaction brought about this classical example of disease burden shift as a result of environmental change, including the influence of alcoholism, smoking and market based food source. ${ }^{40,41}$
Despite of lower prevalence than the general population of year 2015 survey, it was shocking to find all dyslipidaemia cases were newly diagnosed, with $1 / 3$ reported centrally obese. A picture of sedentary lifestyle and unhealthy behaviour increases the odds of dyslipidaemia. Female gender role transition showed significant association with dyslipidaemia, in similar pathogenesis as diabetes. Lim et.al in his systematic review of five large population nutritional studies revealed year 19962006, the era Malaysia was made known to the world has recorded the most rapid rise of our country's population obesity prevalence, proving that modernization is related to the development of obesity. ${ }^{26}$ 
Table III: Factors associated with Hypertension, diabetes mellitus, dyslipidaemia (Simple Logistic Regression Analysis)

\begin{tabular}{|c|c|c|c|c|c|c|c|c|c|}
\hline \multirow{2}{*}{\multicolumn{2}{|c|}{ Variable }} & \multicolumn{3}{|c|}{ Hypertension } & \multicolumn{3}{|c|}{ Diabetes Mellitus } & \multicolumn{2}{|c|}{ Dyslipidaemia } \\
\hline & & $\begin{array}{c}\text { Crude } \\
\text { OR }\end{array}$ & $\begin{array}{c}\text { (95\% Cl } \\
\text { OR) }\end{array}$ & $\begin{array}{c}\mathrm{P} \\
\text { value }^{\mathrm{a}}\end{array}$ & $\begin{array}{c}\text { Crude } \\
\text { OR }\end{array}$ & (95\% CI OR) & P value & Crude OR & (95\% Cl OR) \\
\hline \multicolumn{10}{|c|}{ Socio-demographic risk factor (s) } \\
\hline \multicolumn{10}{|l|}{ Age (year) } \\
\hline & Elderly & 3.171 & $\begin{array}{c}0.939 ; \\
10.713)\end{array}$ & 0.057 & 0.782 & $(0.1923 .188)$ & 0.728 & 0 & $(0.000 ;-)$ \\
\hline & Adult & 1 & & & 1 & & & 1 & \\
\hline \multicolumn{10}{|l|}{ Gender } \\
\hline & Male & 1.376 & $\begin{array}{l}(0.528 ; \\
3.592)\end{array}$ & 0.514 & 0.518 & $(0.162 ; 1.66)$ & 0.257 & 0.259 & $(0.031 ; 2.179)$ \\
\hline & Female & 1 & & & 1 & & & 1 & \\
\hline \multicolumn{10}{|c|}{ Education level } \\
\hline & Low & 7.15 & $\begin{array}{l}(2.123 ; \\
24.083) \\
\end{array}$ & 0.0004 & 0.853 & $(0.284 ; 2.563)$ & 0.778 & 2.381 & $(0.252 ; 22.512)$ \\
\hline & High & 1 & & & 1 & & & 1 & \\
\hline \multicolumn{10}{|c|}{ Job Energy Level } \\
\hline & Low & 0.425 & $\begin{array}{c}0.163 ; \\
1.109)\end{array}$ & 0.077 & 0.55 & $(0.187 ; 1.1614)$ & 0.274 & 3.444 & $(0.365 ; 32.462)$ \\
\hline & High & 1 & & & 1 & & & 1 & \\
\hline \multicolumn{10}{|c|}{ Marital Status } \\
\hline & married & 2 & $\begin{array}{c}(0.6220 \\
6.447)\end{array}$ & 0.234 & 1.75 & $(0.44 ; 6.963)$ & 0.41 & 1.255 & $(0.131 ; 12.051)$ \\
\hline & \begin{tabular}{|l|} 
Not \\
married \\
\end{tabular} & 1 & & & 1 & & & 1 & \\
\hline \multicolumn{10}{|c|}{ Anthropometrical risk factor (s) } \\
\hline \multicolumn{10}{|l|}{ Obesity } \\
\hline & Obese & 1.667 & $\begin{array}{l}(0.619 ; \\
4.489)\end{array}$ & 0.31 & 4.956 & $(1.594 ; 15.412)$ & 0.05 & 3.286 & $(0.51 ; 21.152)$ \\
\hline & $\begin{array}{l}\text { Non } \\
\text { Obese }\end{array}$ & 1 & & & 1 & & & 1 & \\
\hline \multicolumn{10}{|c|}{ Lifestyle risk factor (s) } \\
\hline \multicolumn{10}{|l|}{ Fiber Intake } \\
\hline & No & 1.116 & $\begin{array}{c}0.363 ; \\
3.427)\end{array}$ & 0.848 & 1.503 & $(0.441 ; 5.122)$ & 0.52 & 0 & $(0.000 ;-)$ \\
\hline & Yes & 1 & & & 1 & & & 1 & \\
\hline \multicolumn{10}{|c|}{ Physically Active (>150min / week) } \\
\hline & No & 0.558 & $\begin{array}{c}0.184 ; \\
1.689) \\
\end{array}$ & 0.293 & 1.577 & $(0.494 ; 5.039)$ & 0.447 & 0 & $(0.000 ;-)$ \\
\hline & Yes & 1 & & & 1 & & & 1 & \\
\hline \multicolumn{10}{|l|}{ Smoking } \\
\hline & Yes & 0.981 & $\begin{array}{l}0.381 ; \\
2.526) \\
\end{array}$ & 0.968 & 0.374 & $(0.125 ; 1.121)$ & 0.075 & 0.169 & $(0.018 ; 1.593)$ \\
\hline & No & 1 & & & 1 & & & 1 & \\
\hline \multicolumn{10}{|l|}{ umption } \\
\hline & Yes & 0.769 & $\begin{array}{c}(0.204 ; \\
2.906)\end{array}$ & 0.697 & 0.259 & $(0.031 ; 2.179)$ & 0.149 & 4.296 & $(0.629 ; 29.365)$ \\
\hline & No & 1 & & & 1 & & & 1 & \\
\hline
\end{tabular}

\footnotetext{
${ }^{a}$ Likelihood Ratio(LR) test
} 
Table IV: Factors associated with Hypertension, diabetes mellitus, dyslipidaemia (Multiple Logistic Regression Analysis)

\begin{tabular}{|c|c|c|c|}
\hline Variable & Adj OR & (95\% Cl OR) & $P$ value ${ }^{a}$ \\
\hline \multicolumn{4}{|l|}{ Hypertension } \\
\hline Education level & 13.379 & $3.158 ; 56.656$ & 0 \\
\hline Job Energy Level & 0.094 & $0.019 ; 0.466$ & 0.001 \\
\hline Physically Active (>150min / week) & 0.173 & $0.034 ; 0.822$ & 0.023 \\
\hline Smoking & 0.258 & $0.064 ; 1.033$ & 0.045 \\
\hline \multicolumn{4}{|c|}{ Hosmer-Lemeshow test $=0.822(p>0.05)$} \\
\hline \multicolumn{4}{|l|}{ Prediction power $58.3 \%$} \\
\hline \multicolumn{4}{|l|}{1 influential outlier 1.25} \\
\hline \multicolumn{4}{|l|}{ Collinearity: $\max$ VIF $2.386(<10)$} \\
\hline \multicolumn{4}{|c|}{ All interaction terms checked, not significant } \\
\hline \multicolumn{4}{|l|}{ Diabetes Mellitus } \\
\hline Gender & 0.147 & $0.025 ; 0.863$ & 0.023 \\
\hline Job Energy Level & 0.087 & $0.014 ; 0.532$ & 0.003 \\
\hline Obesity (BMI, WC) & 7.384 & $1.895 ; 28.767$ & 0.002 \\
\hline \multicolumn{4}{|c|}{ Hosmer-Lemeshow test $=0.776(p>0.05)$} \\
\hline \multicolumn{4}{|l|}{ Prediction power $80.6 \%$} \\
\hline \multicolumn{4}{|l|}{5 influential outliers up to 1.5} \\
\hline \multicolumn{4}{|l|}{ Collinearity: max VIF $2.386(<10)$} \\
\hline \multicolumn{4}{|c|}{ All interaction terms checked, not significant } \\
\hline \multicolumn{4}{|l|}{ Dyslipidaemia } \\
\hline Age (year) & 0 & $(0.000 ;-)$ & 0.008 \\
\hline Gender & 413897396.6 & $(0.000 ;-)$ & 0.002 \\
\hline Physically Active (>150min / week) & 0 & $(0.000 ;-)$ & 0.011 \\
\hline Smoking & 0 & $(0.000 ;-)$ & 0.004 \\
\hline \multicolumn{4}{|c|}{ Hosmer-Lemeshow test $=0.0 .961(p>0.05)$} \\
\hline \multicolumn{4}{|l|}{ Prediction power $97.2 \%$} \\
\hline \multicolumn{4}{|l|}{ no influential outlier } \\
\hline \multicolumn{4}{|l|}{ Collinearity: max VIF $2.386(<10)$} \\
\hline All interaction terms checked, not & & & \\
\hline
\end{tabular}

alikelihood Ratio(LR) test, adj OR = Adjusted OR

As the Jakun orang asli usually have short stature, once obesity develops, its prevalence can rapidly rise to higher than the non-aborigine because they have a more disadvantaged predisposing genetic factor. In wide survey done for prevalence of obesity among the Malaysia orang asli, the prevalence of obesity for proto-Malay tribe were 7.5-10\%, with $1 / 3$ as urban fringe dwellers; the condition is well reflected in our study population too. ${ }^{26}$

In examining the role of lifestyle risks in association with non-communicable diseases, harmful lifestyles such as alcohol consumption and smoking conversely related to the disease, while physical activity inversely related to it, fibre intake did not show significant correlation. The type and diversity of fiber was not mentioned, a misclassification of fiber may occur. Monicao et al. 1999 study using Food Frequency Questionnaire in Japanese Brazillian population found no specific relationship of any specific nutrient with glucose intolerance ${ }^{42,43,44}$ while Tsunehara \& Beck-Nielson reported high-fat western diet may related to $\mathrm{DM}$ in contrast. ${ }^{45,46} \mathrm{FFQ}$ is impractical to be administered to aborigine population. Interpreting relationship of diet and 
disease is complicated, confounded by the energy requirement by different sociodemographic characteristics, genetic predisposition for substrate metabolism and physical activity. Low dietary diversity was practiced by the orang asli as mentioned by Haemamalar et al.2010 too. ${ }^{47}$ The Jakun orang asli in Tasik Chini is suffering from the double burden of malnutrition.

Physical activity remained a protective factor from the development of all three non-communicable diseases. Orang asli who are not particular with "time sense", hence daily activities at work and travelling are used as main physical activities, as the orang asli neither specifically perform exercise nor do they practice looking at watch for time. ${ }^{48}$ The Jakun orang asli in Tasik Chini was still considered more active than the general population.

For harmful lifestyles such as smoking and alcohol consumption, more than half of the adult population were smokers, alcohol consumption is twice as high as the national prevalence in 2015 NHMS survey. Smoking was significantly associated with hypertension after adjusted, non-smoking served as protective factor from dyslipidaemia; while alcohol consumption posted a high odd of prevalence odd ratio with diabetes. This is a hint that the population is entering into the transition of modernization in lifestyle, yet still being displaced to a much lower socioeconomy and sociodemographic status, enlarging the gap of social inequity of the community, which can lead to worse health outcome, similarly to the Torres Islander in Australia. ${ }^{26,35,49}$ Remedial action particularly health education should be tailored to the need and understanding level of the Jakun orang asli to divert the practice of unhealthy modifiable risk factors in order to halt the progression of non-communicable diseases.

\section{Strength and Limitation of Study:}

The strength of our study is examining the three cardiovascular risk factors together, this is timely as cardiovascular event has become the leading cause of premature death of non-communicable diseases in Malaysia and worldwide. ${ }^{6,9}$ The study population was from a homogenous Jakun sub-tribe aborigine, where location has been an interest of study for the effect of recent year's urbanization. The study would be useful to contribute to public health program strategy planning. Empowering the orang asli in the healthcare screening and improve education level is a good strategy to create awareness of the disease burden. ${ }^{50}$ Besides, the study demonstrated possible effect of land use and development in relation to the rising burden of noncommunicable diseases should alert the policy maker in re-strategizing the development of this biosphere reserve area.

There are a few limitations in this study, including a small sample size due to case report analysis. There may be information bias based on the category of non-communicable diseases particularly diabetes mellitus when impaired glucose tolerance range is taken as diabetes mellitus, giving rise to a much higher prevalence of diabetes, but this is comparable to the NHMS 2006, 2011, 2015 studies. Recall bias can occur when interviewing orang asli who are more remote and shy in nature in giving information, especially regarding diet history. Future research may look into the effect of urbanization effect to orang asli's noncommunicable diseases development, quantify on fiber intake, physical activity, quantity of alcohol consumption and quantity of smoking; include qualitative method in research may help to further analyze the situation of the studied population; or collaborating with environmental study to examine on the environment and NCD interaction in more defined studies.

\section{CONCLUSION}

In conclusion, the prevalence of hypertension and diabetes mellitus are higher than the national prevalence; majority was undiagnosed. Higher prevalence of unhealthy lifestyles was found. Lifestyle risk factors, obesity and lower sociodemographic status are associated with these non-communicable diseases, yet displacing the orang asli to a much inequitable and disadvantage position. Reduction of modifiable risk factors of non -communicable diseases is vital to halt the progression of the endemic.

\section{Conflict of interest}

The authors declare no conflict of interest with any party. 


\section{ACKNOWLEDGEMENTS}

The authors would like to express their gratitude to the Director General of Health Malaysia for the contribution of data of Ministry of Health; to Dato. Dr. Zainal Ariffin Bin Omar, the initiator of CHINTA (Chini Health Initiative Network Team Approach) project who inspired the survey; to Dr. Mohd Raja Azim Bin Mohd Raja Haron, Public Health physician and Epidemiology Intelligence Officer who led the original survey. Our heartfelt gratitude to Associate Professor Dr. Azmi Bin Mohd Tamil for the proofchecking of statistical data. Highest appreciation is to Pekan District Health officer Dr. Siti Zubaidah bt Abd Rahman and team and Raub District Health office for the participation in data collection. This study was funded by UKM through GGPM-2016-081

\section{REFERENCES}

1. Jabatan Kemajuan Orang Asli. Program Pembangunan Penempatan Tersusun. 2011.

2. World Health Organization. GLOBAL STATUS REPORT on noncommunicable diseases. 2014;2.

3. World Health Organization. NCD in western pacific region 2012. 2012;3.

4. Anon. Indigenous Health: A Worldwide Focus. Lancet. 2016(388).

5. Institute for Public Health. National Health and Morbidity Survey 2011. Non-communicable Diseases. Malaysia2011. p. 192.

6. Institute for Public Health. National Health and Morbidity Survey 2015. 2015.

7. Malaysia MoH. National Strategic Plan for Non Communicable Diseases. 2010. p. 41.

8. Bujang MA, Ismail M, Mohd Hatta NKB, Othman $\mathrm{SH}$, Baharum N, Mat Lazim SS. Validation of the Malay version of Diabetes Quality of Life (DQOL) questionnaire for adult population with type 2 diabetes mellitus. Malaysian Journal of Medical Sciences; 2017. p. 86-96.

9. Ministry of Health Malaysia. National Strategic Plan for Non Communicable Diseases. 2010.

10. Misra PJ, Mini GK, Thankappan KR. Risk factor profile for non-communicable diseases among Mishing tribes in Assam, India: results from a WHO STEPs survey. Indian J Med Res. 2014;140 (3):370-8.

11. Tan AK, Dunn RA, Samad MI, Feisul MI. Sociodemographic and health-lifestyle determinants of obesity risks in Malaysia. Asia Pac J Public Health. 2011;23(2):192-202.
12. World Health Organization. The WHO STEPwise approach to Surveillance of noncommunicable diseases (STEPS). 2001.

13. World Health Organization. GLOBAL ACTION PLAN FOR THE PREVENTION AND CONTROL OF NONCOMMUNICABLE DISEASES 2013-2020. 2013;55(10).

14. World Health Organization. WHO Expert Committee on Biological Standardization. Sixtyfifth report. World Health Organ Tech Rep Ser. 2015(993):1-262.

15. Murray C. GBD 2015 Risk Factors Collaborators. Global, regional, and national comparative risk assessment of 79 behavioural, environmental and occupational, and metabolic risks or clusters of risks, 1990-2015: a systematic analysis for the Global Burden of Disease Study 2015. Lancet, 2016; 388(10053):1659-1724. Lancet; 2016. p. 1659-724.

16. Malaysia JKOA. Structured Settlements Development Programme. 2017.

17. Moszynski P. Health of indigenous people suffers modern world ills. BMJ. 2007;335(7624):795.

18. Chupil T, Joseph J. Creating Knowledge for Change: A Case Study of Sinui Pai Nanek Sengik's Educational Work with Orang Asli Communities in Malaysia. Indigenous Education Program Case Study. Asian South Pacific Bureau of Adult Education (ASPBAE). 2003;21.

19. Cotter P, Condon J, Barnes T, Anderson I, Smith $\mathrm{L}$, Cunningham T. Do Indigenous Australians age prematurely? The implications of life expectancy and health conditions of older Indigenous people for health and aged care policy. Aust Health Rev. 2012;36(1):68-74.

20. Masron $\mathrm{T}$, Masami $\mathrm{F}$, Ismail N. Orang Asli in Peninsular Malaysia : Population, Spatial Distribution and Socio-Economic Condition. J Ritsumeikan Soc Sci Humanit. 2013;6:75-115.

21. Fix AG. Malayan paleosociology: Implications for patterns of genetic variation among the Orang Asli. American Anthropology. 1995:313-23.

22. Ngui R, Shafie Aziz, Chua KH, al. e. Patterns and Risk Factors of Soil-Transmitted Helminthiasis Among Orang Asli Subgroups in Peninsular Malaysia. The American Journal of Tropical Medicine and Hygiene. Aug 2015;93 (2):361 - 70 .

23. Department of Statistic Malaysia. Statistic of Malaysia. 2017.

24. Habibah A, Hamzah J, Mushirifah I. Sustainable Livelihood of the Community in Tasik Chini 
Biosphere Reserve: the Local Practices.

Malaysia: Journal of Sustainable Development; 2010.

25. Academy of Medicine Malaysia. Clinical Practice Guideline on Obesity. 2004.

26. Lim K. A Review of Adult Obesity Research in Malaysia. The Medical Journal of Malaysia. June 2016;71(Supplement 1):1-5.

27. World Health Organization. WHO classification of Body Mass Index. 2017.

28. Ministry of Health Malaysia. HEALTH INDICATORS 2015. 2015:212.

29. Academy of Medicine Malaysia. Clinical Practice Guideline on Type II Diabetes Mellitus. 2009.

30. Institute for Public Health. National Health and Morbidity Survey 2015 Vol I. Methodology \& General Findings. Methodology and General Findings2015.

31. Cheah W, Lee P, Yaman K, Abdul Wahab R. A Preliminary Study on the Prevalence of Cardiovascular Disease Risk factors in Selected Rural COmmunities in Samarahan and Kuching Division, Sarawak, Malaysia. Malaysian J Med Sci. Apr-Jum 2011;18(2):58-65.

32. Fa'iza Abdullah, Tin Myo Han, Mohd Basri Mat Noc, Mohd Aznan M, Ida Zuriaty Ismaild. Prevalence of Hypertension and Glycaemic Control in Adult Type-2 Diabetes Patients: A Preliminary Retrospective Cohort Study in Kuantan, Pahang, Malaysia. International Journal Medicine of Malaysia. 2017;16(1 (1, June 2017)):120.

33. Sithu Aunga, Musa Ramlib, Rahman A Jamalludinc, Shah M Azarismand, Aszrina. A. Role of coping mechanisms to psychosocial stress in early development of hypertension in young adults: a pilot study. International Medicine Journal of Malaysia. 2016;15 (Supplement Issue).

34. James PH. Stress, salt and hypertension. Soc Sci Med. 1988;26(3):293-302.

35. Linda C. Gallo. Do Psychosocial Factors Contribute to Socioeconomic Health Disparities? Applications of the Reserve Capacity Model. Psychological Science Agenda. 2008.

36. Mente A, O'Donnell MJ, Rangarajan S, McQueen MJ, Poirier P, al. WAe. Association of urinary sodium and potassium excretion with blood pressure. The New England journal of medicine. 2014;371(7):601-11.
37. Rafraf M, Mohamadi E, BP. G. Prevalence of Overall and Abdominal Obesity among Adolescent High School Girls in Tabriz, Iran. International Journal Medicine Malaysia. June 2013;12(1).

38. Nicholas C. Center of Orang Asli Concern. 2017.

39. MOHD SAM S. KAJIAN AMALAN BUDAYA ORANG ASLI SUKU KAUM JAKUN DI KAMPUNG PETA. 2015.

40. Kabwama S, Ndyanabangi S, Mutungi G, Wesonga R, Bahendeka S, Guwatudde D. Alcohol use among adults in Uganda: findings from the countrywide non-communicable diseases risk factor cross-sectional survey. Glob Health Action. 2016;9(1):31302.

41. Thakur JS, Garg R, Narain JP, Menabde N. Tobacco use: a major risk factor for non communicable diseases in South-East Asia region. Indian J Public Health. 2011;55(3):15560.

42. Monica B. Costa, Sandra RG. Ferreira, Laercio J. Franco, Suely GA. Gimeno, Magid Lunis, Group. J-BS. Dietary Pattern in High-risk population for glucose intolerance. Journal of Epidemiology. 1999;10(2 March).

43. Hodge A, Montgomery J, Dowse G, Mavo B, Watt T, Zimmet P. A case-control study of diet in newly diagnosed NIDDM in Wanigela people of Papua New Guinea. Diabetes Care. 1996;19:457-62.

44. Colditz GA MJ, Stampfer MJ, Rosner B, , Willet WC SF. Diet and risk of clinical diabetes in women. Am J Clin Nutr. 1992;55(1018-1023).

45. Tsunehara C LD, Fujimoto WY. Diet of secondgeneration Japanese-American men with and without non-insulin-dependent diabetes. Am J Clin Nutr. 1990;52:731-8.

46. Beck-Nielson H, Pederson, Sorenson N. Effects of dietary changes on cellular insulin binding and in vivo insulin sensitivity. Metabolism; 1980,. Metabolism. 1980;29:482-7.

47. Haemamalar K, Zalilah M, Neng A. Nutritional Status of Orang Asli (Che Wong Tribe) Adults in Krau Wildlife Reserve, Pahang. Mal J Nutr. 2010;16(1):55 - 68.

48. Mohd Sam SA. Kajian Amalan Budaya Orang Asli Suku Kaun Jakun di Kampung Peta. 2015.

49. Theo Vos, Bridget Barker, Stephen Begg, Lucy Stanley, Lopez. AD. Burden of disease and injury in Aboriginal and Torres Strait Islander Peoples: the Indigenous health gap. 
International Journal of Epidemiology. 2008;38:470-7.

50. The Star Online. SK Tasik Chini's meteoric rise. 24 May 2015. 
